\title{
LOCALLY COMPLETE INTERSECTION MULTIPLE STRUCTURES ON SMOOTH ALGEBRAIC CURVES
}

\author{
M. BORATYNSKI
}

(Communicated by Louis J. Ratliff, Jr.)

\begin{abstract}
The aim of this paper is to characterize the class of the locally complete intersection multiple structures on smooth curves contained in a smooth three-dimensional variety.
\end{abstract}

\section{INTRODUCTION}

Let $C$ be a smooth (connected) curve contained in a smooth three-dimensional variety $X$. In [1] Banica and Forster described all the multiple structures on $C$, i.e., the locally Cohen Macaulay curves $\bar{C} \subset X$ such that scheme theoretically $\bar{C} \supset C$ and $|\bar{C}|$-the underlying space of $\bar{C}=|C| . \bar{C}$ is called a quasiprimitive multiple structure on $C$ if for almost all points $x \in C \mathrm{em} \operatorname{dim}_{x} \bar{C}$ $=2$.

The aim of this note is to characterize the quasi-primitive multiple structures that are locally complete intersections (lci). In order to make this paper selfcontained, we present first the above-quoted results of Banica and Forster, which we shall need in the formulation of our result.

In the sequel $C$ will be a smooth curve of a three-dimensional smooth (algebraic) variety $X$.

Definition. A locally Cohen Macaulay (ICM) curve $\bar{C} \subset X$ is called a multiple structure on $C$ if $\bar{C} \supset C$ scheme theoretically and $|\bar{C}|=|C|$.

Let $I$ and $J$ denote the ideal sheaves of $C$ and $\bar{C}$ respectively. For any $i \geq$ 1 we define $J_{i}$ as the minimal ideal sheaf containing $J+I^{i}$, which defines a ICM curve of $X$. Since $J_{i}$ is obtained by removing all the embedded components of $J+I^{i}$, we infer that both considered ideals are[A generically equal. We have $J_{1}=I$ and $J_{i}=J$ for $i \geq t+1$ where $t+1$ is the least $i$ such that $J \supset I^{i}$. Moreover $J_{i} \supset J_{i+1}$ for $i \geq 1$.

We claim that $J_{i} \cdot J_{j} \subset J_{i+j}$. This is of course true in all the points of $C$ where $J_{i}=J+I^{i}$. So the ideal $J_{i} \cdot J_{j}+J_{i+j} / J_{i+j} \subset O_{X} / J_{i+j}$ has a zerodimensional support. Since $O_{X} / J_{i+j}$ is $1 \mathrm{CM}$, this ideal is zero and therefore

Received by the editors November 13, 1989 and, in revised form, May 26, 1990.

1991 Mathematics Subject Classification. Primary 14 H99.

This research was supported by the funds of the Ministry of Education. 
$J_{i} \cdot J_{j} \subset J_{i+j}$. In particular $I J_{i} \subset J_{i+1}$. So for all $i \geq 1, J_{i} / J_{i+1}$ is a sheaf of $O_{X} / I$-modules. Since $O_{X} / J_{i+1}$ is $1 \mathrm{CM}$ and $C$ is smooth, the $O_{X} / I$-modules $J_{i} / J_{i+1}$ are locally free.

The multiplication map $J_{i} \times J_{j} \rightarrow J_{i+j}$ induces a generically surjective map $E_{i} \otimes E_{j} \rightarrow E_{i+j}$ where $E_{i}=J_{i} / J_{i+1}$. In particular, one has morphisms $E_{1}^{\otimes i} \rightarrow$ $E_{i}$, which are also generically surjective.

Definition. With the above notation, a multiple structure on $C \subset X$ is called quasi-primitive if $\operatorname{rank} E_{1}=1$. Note that this implies that $\operatorname{rank} E_{i} \leq 1$ for $i \geq 1$. Actually $\operatorname{rank} E_{i}=1$ for $1 \leq i \leq t$ and $E_{i}=0$ for $i>t$.

Now we can formulate our

Theorem. Let $\bar{C}$ be a quasi-primitive multiple structure on $C \subset X$. Then $\bar{C}$ is lci (i.e., $J$ is locally generated by 2 elements) if and only if the morphisms $E_{i} \otimes E_{t-i} \rightarrow E_{t}$ are isomorphisms for $1 \leq i \leq t-1$.

Remark. If $C$ is projective then $E_{i} \otimes E_{t-i} \rightarrow E_{t}$ is an isomorphism if and only if $\operatorname{deg} E_{i}+\operatorname{deg} E_{t-i}=\operatorname{deg} E_{t}$.

Proof. The problem is local. So we can suppose that $O=O_{X}$ "is" a ring of a sufficiently small affine open neighborhood of a point of $C$. Since $C$ is smooth, the epimorphism $O / J \rightarrow O / I$ whose kernel is the nilpotent ideal $I / J$ splits. Therefore $O / J$ admits a certain $O / I$-module structure and $O / J \simeq O / I \oplus I / J$ as $O / I$-modules.

For every $i \geq 1, J_{i} / J$ becomes an $O / I$-submodule of $O / J$. The exact sequences of $O / I$-modules $0 \rightarrow J_{i+1} / J \rightarrow J_{i} / J \rightarrow E_{i} \rightarrow 0$ split since $E_{i}$ is free. Therefore $O / J \simeq O / I \oplus J / I \simeq O / I \oplus E_{1} \oplus \cdots \oplus E_{t}$ as $O / I$-modules $\left(J_{i}=J\right.$ for $\left.i \geq t+1\right) \quad O / I \oplus E_{1} \oplus \cdots \oplus E_{t}$ carries a multiplicative structure that corresponds to the multiplication on $O / J$. We have

$$
E_{i} \cdot E_{j} \subset \bigoplus_{i+j \leq k \leq t} E_{k} \quad \text { since } J_{i} / J \cdot J_{i} / J \subset J_{i+j} / J \text { and } E_{i} \subset J_{i} / J .
$$

Moreover the composed maps

$$
E_{i} \otimes E_{j} \rightarrow E_{i} \cdot E_{j} \rightarrow \bigoplus_{i+j \leq k \leq t} E_{k} \stackrel{\text { projection }}{\longrightarrow} E_{i+j}
$$

coincide with the previously defined morphisms $E_{i} \otimes E_{j} \rightarrow E_{i+j}$.

Suppose that $J$ is lci. This implies that $O / J$ is Gorenstein. Since $O / J$ is a finite module extension of $O / I$, there exists $\pi^{\prime} \in \operatorname{Hom}_{O / I}\left(O / J, E_{t}\right)$ such that the homomorphism $O / J \rightarrow \operatorname{Hom}_{O / I}\left(O / J, E_{t}\right)$ induced by the bilinear form $(, \quad): O / J \times O / J \rightarrow E_{t}(r, s)=\pi^{\prime}(r s)$ is an isomorphism [2]. Note that $E_{t}$ is a rank 1 free $O / I$-module.

Let $\pi: O / J \rightarrow O / I$ be the projection induced by the decomposition $O / J \simeq$ $O / I \oplus E_{1} \oplus \cdots \oplus E_{t}$. There exists $s \in O / J$ such that $\pi(r)=\pi^{\prime}(s r)$ for all $r \in O / J$. It is easy to see that $s$ is invertible. Therefore we can assume that $\pi^{\prime}=\pi$.

Put $E_{0}=O / I$ and let $\gamma$ denote the isomorphism $\bigoplus_{0 \leq i \leq t} E_{i} \rightarrow$ $\operatorname{Hom}\left(\bigoplus_{0 \leq i \leq t} E_{i}, E_{t}\right)$ induced by the bilinear form $(r, s)=\pi(r \cdot s)$. Let $\gamma_{i j} \in$ $\operatorname{Hom}\left(E_{i}, \operatorname{Hom}\left(E_{j}, E_{t}\right)\right)$ denote the $(i, j)$ th entry of the corresponding matrix. The elements $\gamma_{i, t-i} \quad 0 \leq i \leq t$ are on its second diagonal. The elements below 
the second diagonal are zero since $E_{i} \cdot E_{j}=0$ if $i+j>t$. It follows that $\gamma$ is an isomorphism if and only if $\gamma_{i, t-i}$ is an isomorphism for $0 \leq i \leq t$. So the morphisms $E_{i} \otimes E_{t-i} \rightarrow E_{t}$ are isomorphisms since they are induced by $\gamma_{i, t-i}$.

Suppose now that for $1 \leq i \leq t-1$ the maps $E_{i} \otimes E_{t-i} \rightarrow E_{t}$ are isomorphisms. Then $\gamma$ is an isomorphism since the maps $\gamma_{0 t}: E_{0} \rightarrow \operatorname{Hom}\left(E_{t}, E_{t}\right)$ and $\gamma_{t, 0}: E_{t} \rightarrow \operatorname{Hom}\left(E_{0}, E_{t}\right)$ are obviously isomorphisms. To prove that $J$ is a lci it suffices to invoke that "in codimension 2 case Gorenstein implies complete intersection."

\section{REFERENCES}

1. C. Banica and O. Forster, Multiplicity structures on space curves, The Lefschetz centennial conference, part I, Contemp. Math., vol. 58, Amer. Math. Soc., Providence, RI, 1986, pp. 47-64.

2. R. Hartshorne, Residues and duality, Lecture Notes in Math., vol. 20, Springer-Verlag, Berlin, Heidelberg, New York, 1966.

Dipartimento di Matematica, Universitá degli Studi, via G. Fortunato, 70125 Bari, ITALY 